\title{
SOME RANKS OF MODULES OVER GROUP RINGS
}

\author{
V.A. BOVDI AND L.A. KURDACHENKO \\ Dedicated to Professor Igor Subbotin on the occasion of his $70^{\text {th }}$ birthday
}

\begin{abstract}
A commutative ring $R$ has finite rank $r$, if each ideal of $R$ is generated at most by $r$ elements. A commutative ring $R$ has the $r$-generator property, if each finitely generated ideal of $R$ can be generated by $r$ elements. Such rings are closely related to Prüfer domains. In the present paper we investigate some analogs of these concepts for modules over group rings.
\end{abstract}

\section{INTRODUCTION AND RESULTS}

The concept of dimension of a vector space is one of the most important concepts of mathematics. It is the source of many important numerical invariants in many areas of mathematics.

Modules are natural generalizations of vector spaces, therefore, it is natural to extend the concept of dimension to them. One possibility is the concept of the $R$-rank of an $R$-module $A$, where $R$ is a ring. It is defined as the cardinality of a maximal $R$-free subset of $A$. This definition turned out to be effective only for the case when $A$ is an $R$-torsion free module over an integral domain $R$. Other extensions of the concept of dimension are related to the number of generators. Unlike in the case of vector spaces, not each submodule of a finitely generated module is finitely generated. Moreover, when a submodule is f.g. (finitely generated), it can have a minimal generating set whose cardinality is greater than the number of elements in the generating system of the entire module. The reasons of this phenomena are related to the structure of the ring $R$, over which the module is considered. Not evry left (or right) ideal of a ring is finitely generated. Rings having such property are called Noetherian. However, even in notherian rings the number of generators of left ideals are not always bounded. Following Cohen [5], a commutative ring $R$ has finite rank $r$, if each ideal of $R$ has $r$ generators. Almost at the same time, the concept of special rank arises in group theory (Maltsev [15]). Dedekind and principal ideal domains are examples of such rings. Commutative rings, having finite rank, were studied in [5, 10, 11, 12]. The notion of rings of finite rank was generalized by Gilmer [10]. Following Gilmer, we say that a commutative ring $R$ has an $r$-generator property, if each finitely generated ideal of $R$ can be generated by $r$ elements. Examples of such rings are the Bezout domains with the 1 -generator property. Commutative rings having the $r$-generator property have been actively studied in [10, 11, 12]. In particular, such rings proved to be closely related to Prüfer domains.In our investigation we consider analogs of these concepts for modules over group rings. Since the notion of " $R$-rank" is already used in representation theory, we adapt the terminology from group theory as it was proposed by Maltsev in [15]. A module $A$ over a ring $R$ has special rank $r$ if each f.g. (finitely generated) $R$-submodule of $A$ can be generated by $r$ elements and there exists at least one f.g. $R$-submodule $D$ of $A$, which has a minimal generating subset, consisting exactly of $r$ elements. The special $R$-rank and the classical $R$-rank of $A$ are

2010 Mathematics Subject Classification. Primary: 20C05; 20F50 Secondary: 20D25; 16D10; 16D70; 16D80. Key words and phrases. module over ring; special rank; Prüfer domain; Dedekind domain. 
denoted by $\operatorname{sr}_{R}(A)$ and $\operatorname{rank}_{R}(A)$, respectively. If $\operatorname{sr}_{R}(A)$ is finite, then we write $\operatorname{sr}_{R}(A) \in \mathbb{N}$, where $\mathbb{N}$ is the set of positive integers.

Note that in the theory of groups, the topic related to groups of finite special $F G$-rank have played and continue to play a significant role. A huge array of articles has been devoted to this subject, in which many interesting and profound results have been obtained. An excellent overview of the basic results obtained in this topic is contained in the article of Dixon [8]. The book [9] is devoted to the presentation of many results connected to groups of finite special rank.

The first natural step in our investigation is the case when $R G$ is the group algebra of a finite group $G$ over a field $R$. Our main result is the following.

Theorem 1. Let FG be the group algebra of a finite group $G$ over a field $F$ of characteristic 0 . $A$ right $F G$-module $A$ has a finite special $R$-rank $\operatorname{sr}_{F G}(A)=r \in \mathbb{N}$ if and only if the following conditions hold:

(i) $A=\oplus_{1 \leq j \leq n} H_{j}$ with $H_{j}=\oplus_{1 \leq k \leq t(j)} D_{k}$, where each $D_{k}$ is a simple right $F G$-submodule of $A$ and $D_{1} \cong_{F G} D_{2} \cong_{F G} \cdots \cong_{F G} D_{t(j)}$;

(ii) $t(j) \leq r$ for all $1 \leq j \leq n$ and there exist $s \in \mathbb{N}$ such that $t(s)=r$;

(iii) $n$ is bounded by the number $\operatorname{nns}_{F G}(G)$ of pairwise non-isomorphic simple FG-modules.

In particular, if $\operatorname{sr}_{F G}(A)=r \in \mathbb{N}$, then $\operatorname{dim}_{F}(A) \leq r \cdot|G| \cdot \operatorname{nns}_{F}(G)$.

If $G$ is a finite group and $R$ is an integral domain of characteristic 0 , then a description of $R$-torsion-free $R G$-modules, having finite special rank, is given by the following.

Theorem 2. Let $R G$ be the group ring of a finite group $G$ over an integral domain $R$ of characteristic 0 and let $F$ be the field of fractions of $R$. Let $A$ be a right $R G$-module, which is $R$-torsion free. The $R G$-module $A$ has special rank $\operatorname{sr}_{R G}(A)=r \in \mathbb{N}$ if and only if the right $F G$-module $A \otimes_{R} F$ has the same special rank $\operatorname{sr}_{F G}\left(A \otimes_{R} F\right)=r$. In particular, $\operatorname{rank}_{R G}(A) \leq$ $\operatorname{nns}_{F}(G)$, where $\operatorname{nns}_{F G}(G)$ is the number of pairwise non-isomorphic simple $F G$-modules.

Let $A$ be a right module over an integral domain $R$. If $A$ has a finite $R$-rank, then the section $A / \omega(R G) A$ also has a finite $R$-rank, where $\omega(R G)=\langle g-1 \mid g \in G\rangle_{R}$ is the augmentation ideal of $R G$. Since the action of $G$ on the right module $A / \omega(R G) A$ is trivial, $A / \omega(R G) A$ is an $R$-module. Therefore, it is natural to consider modules over $R G$ having finite special ranks in which $R$ is a commutative ring with the $r$-generator property. An important example is the case when $R$ is a Dedekind domain. Our next result is the following.

Theorem 3. Let $D G$ be the group ring of a finite group $G$ over a Dedekind domain $D$, all whose capitals have characteristic 0 . Let $F$ be the field of fractions of the ring $D$. Let $A$ be a right $D G$-module which is $D$-periodic. The module $A$ has special rank $\operatorname{sr}_{D G}(A)=r \in \mathbb{N}$ if and only if the following conditions hold:

(i) $A=\oplus_{P \in \pi} A_{P}$ where $\pi=\operatorname{Ass}_{R}(A)$ and $A_{P}$ is the $P$-component of $A$;

(ii) $A_{P}=\oplus_{1 \leq j \leq n(P)} H_{j, P}$ where $n(P) \leq \operatorname{nns}_{F}(G)$ and each $H_{j, P}$ is a $G$-homogeneous DGsubmodule of $A$ and it is Artinian as a $D$-module;

(iii) $\Omega_{[P, 1]}\left(H_{j, P}\right)$ is a direct sum of at most $r$ simple $D G$-submodules of $A$;

(iv) there exist $t \in \mathbb{N}$ and a maximal ideal $P$ such that $\Omega_{[P, 1]}\left(H_{t, P}\right)$ is a direct sum of $r$ copies of simple DG-submodules of $A$.

All necessary definitions and notations are given in the next section. 


\section{PReliminaries AND LEMmas}

In the sequel, each module means a right module, unless otherwise specified. Let $F G$ be the group algebra of a finite group $G$ over a field $F$ of characteristic 0. Each $F G$-module $A$ is semisimple (see [13, Corollary 5.15]), so the algebra $F G$ is semisimple too. The number of pairwise non-isomorphic simple $F G$-modules is denoted by $\operatorname{nns}_{F}(G)$. When $F G$ is a finite dimensional $F$-algebra, $\operatorname{nns}_{F}(G)$ is a finite number (see [1, 2]) which was calculated in the following way: Let $\xi$ be a primitive $n$-th root of unity, where $n$ is the greatest divisor of $\exp (G)$ which is not divisible by $\operatorname{char}(F)$. Two elements $a, b \in G$ are called $F$-conjugate if there exist $x \in G$ and $m \in \mathbb{N}$ such that $x^{-1} a x=b^{m}$ and the map $\xi \mapsto \xi^{m}$ is extensible to an automorphism of the field $F(\xi)$ fixing the subfield $F$ elementwise.

Berman [2] and Witt [16] proved that $\operatorname{nns}_{F}(G)$ is equal to the number of $F$-conjugate classes of $F$-regular elements of $G$ (see [7, Theorem 42.8 p. 306]). If $F$ is algebraically closed, then Brauer [4, 7] shows that $\operatorname{nns}_{F}(G)$ is equal to the number of the conjugate classes of elements of $G$ (see [7, Theorem 40.1 p. 283]). In both cases $n s_{F}(G)$ is bounded by the number of conjugacy classes of $G$.

Let $A$ be a module over an integral domain $R$ and let $G$ be a finite group. The set

$$
\operatorname{Ass}_{R}(A)=\left\{P \mid P \quad \text { is a prime ideal of } R \text { such that } \quad \operatorname{Ann}_{A}(P) \neq\langle 0\rangle\right\}
$$

denotes the $R$-assassinator of $A$. The $R$-submodule

$$
A_{U}=\left\{a \in A \mid a U^{n}=\langle 0\rangle \quad \text { for some } \quad n \in \mathbb{N}\right\}
$$

of $A$ is called the $U$-component of $A$. If $A=A_{U}$, then $A$ is called the $U$-module.

For each $U$-module $A$ and each $k \in \mathbb{N}$ we define the following $R$-submodules of $A$ :

$$
\Omega_{[U, k]}(A)=\left\{a \in A \mid a U^{k}=\langle 0\rangle\right\} \quad \text { and } \quad A_{U}=\cup_{s \in \mathbb{N}} \Omega_{[U, s]}(A) .
$$

Obviously, $\quad \Omega_{[U, 1]}(A) \leq \Omega_{[U, 2]}(A) \leq \cdots \leq A_{U}$.

Let $A$ be a $D G$-module such that $A$ is a $P$-module for some maximal ideal $P$ of a Dedekind domain $D$. The module $A$ is called $(G, P)$-homogeneous if $A$ has an ascending series of $D G$ submodules whose factors are isomorphic as $D G$-modules. A capital of a Dedekind domain $D$ is a factor-ring $D / P$ in which $P$ is a maximal ideal of $D$.

Let $A$ and $B$ be $R$-modules over an integral domain $R$. The notation $A_{R}$ (or $\left.(A)_{R}\right)$ denotes the fact that $A$ is an $R$-module. Define the following $R$-submodule:

$$
\operatorname{Tor}_{R}(A)=\left\{a \in A \mid \operatorname{Ann}_{R}(a) \neq\langle 0\rangle\right\} \leq A_{R} .
$$

We denoted the annihilator and the left annihilator of a module $A$ by $\operatorname{Ann}_{R}(A)$ and $\operatorname{Ann}_{R}^{\text {left }}(A)$, respectively. The $R$-submodule of $A$ generated by the elements $a_{1}, \ldots, a_{n} \in A$ is denoted by $\left\langle a_{1}, \ldots, a_{n}\right\rangle_{R}$. The $R$-isomorphism between $R$-modules $A$ and $B$ is denoted by $A \cong_{R} B$.

The module $A$ is called periodic as an $R$-module (or simply, $R$-periodic), if $\operatorname{Tor}_{R}(A)=A$. In other words, $A$ is $R$-periodic if $\operatorname{Ann}_{R}(a) \neq\langle 0\rangle$ for each $a \in A$. If $\operatorname{Tor}_{R}(A)=\langle 0\rangle$, then we say that $A$ is $R$-torsion-free. The intersection $\Phi(A)$ of all maximal $R$-submodules of $A$ is called the Frattini submodule of $A$. Of course, if $A$ does not include proper maximal submodules, then $\Phi(A)=A$.

In the sequel, we use the notions and results from the books [3, 7, 9].

We start our proof with the following. 
Lemma 1. Let $A$ be an $R$-module over a ring $R$ such that $\operatorname{sr}_{R}(A)$ is finite. Let $B$ and $C$ be $R$-submodules of $A$ such that $B \subseteq C$. The following conditions hold:

(i) $\operatorname{sr}_{R}(B) \leq \operatorname{sr}_{R}(C) \leq \operatorname{sr}_{R}(A)$;

(ii) $\operatorname{sr}_{R}(C / B) \leq \operatorname{sr}_{R}(A / B) \leq \operatorname{sr}_{R}(A)$.

These assertions are obvious.

Lemma 2. Let $B$ be an $R$-submodule of an $R$-module $A$ over a Noetherian ring $R$. If $\operatorname{sr}_{R}(B)$ and $\operatorname{sr}_{R}(A / B)$ are finite numbers, then $\operatorname{sr}_{R}(A) \leq \operatorname{sr}_{R}(B)+\operatorname{sr}_{R}(A / B)$.

Proof. Let $D$ be a f.g. $R$-submodule of $A$. Clearly, $D /(D \cap B) \cong_{R}(D+B) / B$ has a finite generating subset $\left\{d_{j}+(D \cap B) \mid 1 \leq j \leq m\right\}$ with $m \leq \operatorname{sr}_{R}(A / B)$. Since $D$ is a f.g. module over a Noetherian ring, $D$ is a Noetherian $R$-module (see [13, Lemma 1.1]), so $D \cap B$ is a f.g. $R$-submodule of $B$. Since $\operatorname{sr}_{R}(B)$ is finite, there exists a finite set of generators $\left\{b_{j} \mid 1 \leq j \leq k\right\}$ of $D \cap B$ with $k \leq \operatorname{sr}_{R}(B)$. The subset $\left\{d_{j} \mid 1 \leq j \leq m\right\} \cup\left\{b_{j} \mid 1 \leq j \leq k\right\}$ generates $D$ as an $R$-submodule and $m+k \leq \operatorname{sr}_{R}(B)+\operatorname{sr}_{R}(A / B)$.

Lemma 3. Let $A=\oplus_{1 \leq j \leq n} A_{j}$ be an $R$-module over a ring $R$ in which each $A_{j}$ is a simple $R$-submodule. If $\operatorname{Ann}_{R}^{\text {left }}\left(A_{j}\right) \neq \operatorname{Ann}_{R}^{\text {left }}\left(A_{i}\right)$ for all $i \neq j$, then $A$ is a cyclic $R$-module.

Proof. Since each $A_{j}$ is a simple $R$-submodule, $U_{j}=\operatorname{Ann}_{R}^{\text {left }}\left(A_{j}\right)$ is a maximal left ideal of $R$. From $U_{i} \neq U_{j}$ follows that $U_{i}+U_{j}=R$ for all $i \neq j$.

Let us prove that $A=\left\langle a_{1}+\cdots+a_{n} \mid 0 \neq a_{i} \in A_{i}\right\rangle_{R}$. Indeed, $U_{1}+U_{2}=R$ shows that we can choose $\alpha_{1} \in U_{2}$ such that $\alpha_{1} \notin U_{1}$. Clearly $\alpha_{1} a_{1} \neq 0, \alpha_{1} a_{2}=0$ and

$$
\alpha_{1}\left(a_{1}+a_{2}+\cdots+a_{n}\right)=\alpha_{1} a_{1}+\alpha_{1} a_{3}+\cdots+\alpha_{1} a_{n} .
$$

If $\alpha_{1} a_{3}+\cdots+\alpha_{1} a_{n}=0$, then $\alpha_{1} a_{1} \in R\left(a_{1}+\cdots+a_{n}\right)$. Since $A_{1}$ is a simple $R$-submodule, it is generated by any non-zero element, so $A_{1}=R a_{1} \leq R\left(a_{1}+\cdots+a_{n}\right)$.

Assume now that $\alpha_{1} a_{3}+\cdots+\alpha_{1} a_{n} \neq 0$. Let $j_{1}$ be a smallest positive integer (say, $j_{1}=3$ ), such that $j_{1}>2$ and $\alpha_{1} a_{j_{1}} \neq 0$. The equality $U_{1}+U_{3}=R$ shows that we can choose $\alpha_{2} \in U_{3}$ with $\alpha_{2} \notin U_{1}$. Hence $\alpha_{2} \alpha_{1} a_{1} \neq 0, \alpha_{2} \alpha_{1} a_{3}=0$ and

$$
\alpha_{2}\left(\alpha_{1} a_{1}+\alpha_{1} a_{3}+\cdots+\alpha_{1} a_{n}\right)=\alpha_{2} \alpha_{1} a_{1}+\alpha_{2} \alpha_{1} a_{4}+\cdots+\alpha_{2} \alpha_{1} a_{n} .
$$

If $\alpha_{2} \alpha_{1} a_{4}+\cdots+\alpha_{2} \alpha_{1} a_{n}=0$ then, as above, we have $A_{1}=R a_{1} \leq R\left(a_{1}+\cdots+a_{n}\right)$. Otherwise, we can repeat finitely many times the mentioned argument above. Finally we obtain that $A_{1} \leq R\left(a_{1}+\cdots+a_{n}\right)$. Similarly, using the same argument, $A_{j}=R a_{j} \leq R\left(a_{1}+\cdots+a_{n}\right)$ for each $1 \leq j \leq n$, so $A=A_{1} \oplus \cdots \oplus A_{n}=R\left(a_{1}+\cdots+a_{n}\right)$.

Lemma 4. Let $A=\oplus_{1 \leq j \leq n} A_{j}$ be an $R$-module over a ring $R$, in which each $A_{j}$ is a simple $R$-submodule. If

$$
\operatorname{Ann}_{R}^{\text {left }}\left(A_{1}\right)=\operatorname{Ann}_{R}^{\text {left }}\left(A_{2}\right)=\cdots=\operatorname{Ann}_{R}^{\text {left }}\left(A_{n}\right),
$$

then $A_{j} \cong_{R} R a$ for each $0 \neq a \in A$ and $1 \leq j \leq n$.

Proof. Let $a=a_{1}+\cdots+a_{n} \in A$ where each $a_{j} \in A_{j}$ for $1 \leq j \leq n$. Without loss of generality, we can assume $a_{1} \neq 0$. Put $B=R a$ and $D=A_{2} \oplus \cdots \oplus A_{n}$. Then $(B+D) / D$ is a non-zero submodule of $A / D$. The isomorphism $A / D \cong_{R} A_{1}$ shows that $A / D$ is a simple $R$ - module. It follows that either $A / D=(B+D) / D$ or $A=B+D$. Using the isomorphism $(B+D) / D \cong_{R} B /(B \cap D)$, we obtain that $B /(B \cap D) \cong_{R} A_{1}$. Suppose that $B \cap D \neq 0$ and choose $0 \neq c \in B \cap D$. Since $c \in B=R a$ and $c=y a$ for some $y \in R, c=y a_{1}+\cdots+y a_{n}$. 
On the other hand, $c \in D$, so that $p r_{1}(c)=y a_{1}=0$ in which $\operatorname{pr}_{j}(A)=A_{j}$. It follows that $y \in \operatorname{Ann}_{R}^{\text {left }}\left(a_{1}\right)$. Since $A_{1}$ is a simple $R$-submodule and $a_{1} \neq 0, \operatorname{Ann}_{R}^{\text {left }}\left(a_{1}\right)=\operatorname{Ann}_{R}^{\text {left }}\left(A_{1}\right)$. This yields $y \in \operatorname{Ann}_{R}^{\text {left }}\left(A_{j}\right)$ for each $1 \leq j \leq n$. Consequently, $y a_{j}=0$ for all $j$, so $c=0$ is a contradiction. It proves that $B \cap D=\langle 0\rangle$ and $B \cong_{R} A_{1}$.

Corollary 1. Let $A=\oplus_{\lambda \in \Lambda} A_{\lambda}$ be an $R$-module over a ring $R$ in which each $A_{\lambda}$ is a simple $R$-submodule, where $\Lambda$ is a linearly ordered set. If $\operatorname{Ann}_{R}^{\text {left }}\left(A_{\lambda}\right)=\operatorname{Ann}_{R}^{\text {left }}\left(A_{\mu}\right)$ for all $\lambda, \mu \in \Lambda$, then $R a \cong_{R} A_{\delta}$, for each $0 \neq a \in A$ and $\delta \in \Lambda$.

Corollary 2. Let $A=\oplus_{1 \leq j \leq n} A_{j}$ be an $R$-module over a ring $R$ in which each $A_{j}$ is a simple $R$-submodule. If $A_{1} \cong_{R} A_{2} \cong_{R} \cdots \cong_{R} A_{n}$, then $\operatorname{sr}_{R}(A)=r \in \mathbb{N}$ if and only if $n=r$.

Proof. Let $B=\langle S\rangle_{R}$ be a f.g. $R$-submodule of $A$ with a minimal generating subset $S=$ $\left\{b_{1}, \ldots, b_{k}\right\}$. The $R$-submodule $B_{1}=\left\langle b_{1}\right\rangle_{R} \cong_{R} A_{1}$ by Lemma 4. Since $B$ is a semisimple $R$-module (see [13, Corollary 4.4]), there exists an $R$-submodule $D_{1}$ such that $B=B_{1} \oplus D_{1}$. Clearly $b_{2} \in D_{1}$ and $b_{2} \notin B_{1}$, by the minimality of $S$. The $R$-submodule $B_{2}=\left\langle b_{2}\right\rangle_{R}$ is isomorphic to $A_{1}$ by Lemma 4. Repeating this argument finitely many times, we obtain a direct decomposition $B=B_{1} \oplus B_{2} \oplus \cdots \oplus B_{k}$, where each $B_{j} \cong_{R} A_{1}$.

Choosing a different minimal generating subset $\left\{d_{1}, \ldots, d_{m}\right\}$ of $B$ and repeating the same argument, we construct a direct decomposition $B=D_{1} \oplus \cdots \oplus D_{m}$ in which each $D_{j} \cong_{R} A_{1}$. Clearly $k=m$ by the Krull-Remak-Schmidt theorem (see [6, Chapter 6, Theorem 1.6]). In particular, if $B=A$, then $n \leq r$ and the above argument shows that $n=r$.

Let $A$ be an $R$-module over a ring $R$, such that $A=\oplus_{1 \leq j \leq n} A_{j}$ in which each $A_{j}$ is a simple $R$-submodule. The relation " $A_{i} \cong_{R} A_{j}$ " is an equivalence relation on the set $\left\{A_{j} \mid 1 \leq j \leq n\right\}$ with equivalence classes $E_{1}, \ldots, E_{k}$. The direct sum of all submodules from the set $E_{j}$ is denoted by $S_{j}$ and it is called the homogeneous component of $A$.

Corollary 3. Let $A=\oplus_{1 \leq j \leq n} A_{j}$ be an $R$-module over a ring $R$, in which each $A_{j}$ is a simple $R$-submodule and let $E_{1}, \ldots, E_{k}$ be the homogeneous components of $A$. The number $r=\operatorname{sr}_{R}(A)$ is finite if and only if the following conditions hold:

(i) $\operatorname{sr}_{R}\left(E_{j}\right) \leq r$ for each $j \in\{1, \ldots, k\}$;

(ii) there exist at least one $j \in\{1, \ldots, k\}$ such that $\operatorname{sr}_{R}\left(E_{j}\right)=r$.

Proof. Clearly $A=E_{1} \oplus \cdots \oplus E_{k}$, where each $E_{j}=A_{j, 1} \oplus \cdots \oplus A_{j, t(j)}$ has a special rank $\operatorname{sr}_{R}\left(E_{j}\right)=t(j) \leq r$ by Corollary 2, Set $s=\max \{t(1), \ldots, t(k)\}$. It is easy to see that each $B_{i}=\oplus_{1 \leq m \leq k} A_{m, i}$ is cyclic by Lemma 3. It follows that $A$ has a minimal generating subset, consisting exactly of $s$ elements. Moreover, any $R$-submodule $D \leq A$ is also semisimple, so $D=\oplus_{1 \leq l \leq q} D_{l}$ in which $q \leq n$. Furthermore, each simple $R$-submodule $D_{l}$ is isomorphic to a submodule from the set $\left\{A_{1}, \ldots, A_{n}\right\}$ (see [13, Corollary 4.4]). Repeating the above arguments for $D$, we obtain that the minimal generating subset of $D$ consists at most of $s$ elements, so $s=r$.

\section{Proofs}

Proof of Theorem 1. The fact $\operatorname{char}(F)=0$ implies that $A$ is a semisimple $F G$-module (see [13. Corollary 5.15]), so $A=\oplus_{1 \leq j \leq n} H_{j}$ in which each $H_{j}$ is a homogeneous component of $A$ and $H_{j}$ is a direct sum of at most $r$ simple submodules by Corollary 2, As we noted above, $n \leq \operatorname{nns}_{F}(G)$. Obviously, the dimension of a simple $F G$-submodule of $A$ is bounded by $|G|$, 
so $\operatorname{dim}_{F}\left(H_{j}\right) \leq r|G|$ and $\operatorname{dim}_{F}(A) \leq r \cdot|G| \cdot \operatorname{nns}_{F}(G)$. The sufficiency of the conditions of our Theorem follows from Corollary 3 .

Proof of Theorem Q Put $B=A \otimes_{R} F$. Let $C=\left\langle b_{1}, \ldots, b_{n}\right\rangle_{F G}$ be an $F G$-submodule of $B$ generated by the subset $\left\{b_{1}, \ldots, b_{n}\right\} \subseteq A$. For each $b_{j}$ there exists a non-zero $y_{j} \in R$ such that $a_{j}=y_{j} b_{j} \in A$. This choice ensures that the $F G$-submodule $\left\langle a_{1}, \ldots, a_{n}\right\rangle_{F G}$ of $B$ coincides with $C$. Let $E=\left\langle a_{1}, \ldots, a_{n}\right\rangle_{R G}$ be an $R G$-submodule. Clearly, $C / E$ is an $R$-periodic $R$-module. The fact that $A$ has a finite special rank $r$ implies that $E$ has an $R G$-generating subset $e_{1}, \ldots, e_{r}$ in which $r \leq n$. The construction of $E$ implies that $C=\left\langle e_{1}, \ldots, e_{r}\right\rangle_{F G}$. Thus each finitely generated $F G$-submodule of $B$ can be generated by at most $r$ elements. It follows that the $F G$-module $B$ has a finite special rank at most $r$. Moreover, using similar arguments and Theorem 1, we can prove that $B$ has a special rank $r$ and $B$ has a finite dimension $d$ at most $r|G| \operatorname{nns}_{F}(G)$. Let $\left\{v_{1}, \ldots, v_{d}\right\}$ be a basis of B. Again, for each $v_{j}$ we can choose a non-zero $z_{j} \in R$ such that $u_{j}=z_{j} v_{j} \in A$. Since $\left\{v_{1}, \ldots, v_{d}\right\}$ is a basis of $B,\left\{u_{1}, \ldots, u_{d}\right\}$ is a maximal independent subset of $A$. Consequently, the $R$-rank of $A$ is exactly $d$.

Lemma 5. Let $D G$ be the group ring of a finite group $G$ over a Dedekind domain $D$ of characteristic 0 . Let $A$ be a $D G$-module which is also a $P$-module for some maximal ideal $P$ of $D$ with the property that char $(D / P)=0$. If $B$ is a $D G$-submodule of $A$ such that $A=B \oplus C$ for a $D$-submodule $C$ of $A$, then there exists a $D G$-submodule $K$ with the property that $A=B \oplus K$.

Proof. Put $D_{P}=D / P$. Consider the $D$-submodule $\Omega_{[P, 1]}(A)$ (see (1)). It is easy to see that $\operatorname{Ann}_{D}(a)=P$ for each $a \in \Omega_{[P, 1]}(A)$, so $\Omega_{[P, 1]}(A)$ can be considered as a $(D / P) G$-module. In particular, the additive group of $\Omega_{[P, 1]}(A)$ is a direct sum of isomorphic copies of the additive group $D / P$. Since $\operatorname{char}(D / P)=0$, the additive group of $D / P$ is divisible, so the additive group of $\Omega_{[P, 1]}(A)$ is divisible too. Also the additive group of $\Omega_{[P, j+1]}(A) / \Omega_{[P, j]}(A)$ is divisible for each $j$ by the same reason, so the additive group of $A=\cup_{j \in \mathbb{N}} \Omega_{[P, j]}(A)$ has an ascending series whose factors are divisible. It follows that the additive group of $A$ is divisible. There exists a $D G$-submodule $K$ such that $n A \leq B+K$ and $n(B \cap K)=\langle 0\rangle$ by [13, Theorem 5.9]. The fact that the additive group of $A$ is divisible implies that $n A=A$, so $A=B+K$. The fact that $\operatorname{char}(D / P)=0$ implies that an additive group of $A$ is torsion-free, so that $B \cap K=\langle 0\rangle$ and $A=B \oplus K$.

Lemma 6. Let $D G$ be the group ring of a finite group $G$ over a Dedekind domain $D$ of characteristic 0 . Let $A$ be a $D G$-module such that $A$ is also a $P$-module for some maximal ideal $P$ of $D$. If $\operatorname{char}(D / P)=0$ and $\operatorname{sr}_{D G}(A)=r \in \mathbb{N}$, then $A$ is an Artinian D-module.

Proof. Put $D_{P}=D / P$. The $D$-submodule $\Omega_{[P, 1]}(A)$ is a $D G$-submodule of $A$ and $\operatorname{Ann}_{D}(a)=P$ for each $a \in \Omega_{[P, 1]}(A)$. Obviously, $\Omega_{[P, 1]}(A)$ is a $D_{P}[G]$-module and $\operatorname{dim}_{D_{P}}\left(\Omega_{[P, 1]}(A)\right)$ is finite by Lemma 1 and Theorem 1. Thus $A$ is Artinian as a $D$-module (see [14, Corollary 7.12]).

Let $A$ be a simple $R$-module over a Dedekind domain $R$ (in particular, principal ideal domain). Clearly, $A \cong R / P$ for some maximal ideal $P$. The factors $R / P^{k}$ and $P / P^{k+1}$ are isomorphic as $R$-modules for each $k \in \mathbb{N}$ (see [14, Corollary 1.28]). In particular, the $R$-module $R / P^{k}$ is embedded in the $R$-module $R / P^{k+1}$ for each $k \in \mathbb{N}$ and $\left\{R / P^{k} \mid k \in \mathbb{N}\right\}$ is an injective family of $R$-modules. Consider the $R$-module $C_{P^{\infty}}=\lim _{\text {inj }}\left\{R / P^{k} \mid k \in \mathbb{N}\right\}$ which is called the Prüfer $P$-module. It is easy to see that $C_{P \infty}$ is a $P$-module, $\Omega_{[P, k]}\left(C_{P \infty}\right) \cong_{R} R / P^{k}$ and

$$
\Omega_{[P, k+1]}\left(C_{P^{\infty}}\right) / \Omega_{[P, k]}\left(C_{P^{\infty}}\right) \cong_{R}\left(R / P^{k+1}\right) /\left(P / P^{k+1}\right) \cong_{R} R / P .
$$


Hence, if $C$ is a proper $R$-submodule of $C_{P^{\infty}}$ (i.e. $\left.C \neq C_{P^{\infty}}\right)$, then $C=\Omega_{[P, k]}\left(C_{P^{\infty}}\right)$ for some $k \in \mathbb{N}$. Similarly, if $b \notin \Omega_{[P, k-1]}\left(C_{P^{\infty}}\right)$, then $C=R b$. Observe also that a Prüfer $P$-module is monolithic and its monolith coincides with $\Omega_{[P, 1]}\left(C_{P \infty}\right)$. Indeed, the intersection $C \cap \Omega_{[P, 1]}\left(C_{P \infty}\right)$ is non-zero for each $R$-submodule $C$ of a Prüfer $P$-module. Since $\Omega_{[P, 1]}\left(C_{P \infty}\right)$ is a simple $R$ module $\left(\Omega_{[P, 1]}\left(C_{P \infty}\right)\right.$ is isomorphic to $\left.R / P\right)$,

$$
C \cap \Omega_{[P, 1]}\left(C_{P^{\infty}}\right)=\Omega_{[P, 1]}\left(C_{P^{\infty}}\right) .
$$

It follows that the $R$-monolith of a Prüfer $P$-module includes $\Omega_{[P, 1]}\left(C_{P \infty}\right)$ and, hence coincides with it, because $\Omega_{[P, 1]}\left(C_{P \infty}\right)$ is a simple $R$-submodule.

Indeed, let $C$ be a finitely generated $R$-submodule of a Prüfer $P$-module. Then $\mathrm{C}$ is a proper $R$-submodule. As we have seen above, $C$ coincides with $\Omega_{[P, n]}\left(C_{P \infty}\right)$ for some $n \in \mathbb{N}$. The last $R$-submodule is cyclic, it is isomorphic to $R / P^{n}$. Hence each f.g. $R$-submodule of a Prüfer $P$-module is cyclic. It follows that a Prüfer $P$-module has $R$-rank 1 .

Corollary 4. Let $D G$ be the group ring of a finite group $G$ over a Dedekind domain $D$. Let $A$ be a $D G$-module of $\operatorname{sr}_{D G}(A)=r \in \mathbb{N}$ such that it is also a $P$-module for some maximal ideal $P$ of $D$. If $\operatorname{char}(D / P)=0$, then $A=K \oplus B$ in which $K, B$ are $D G$-submodules. Moreover, $K$ is a f.g. D-submodule and $B$ is a direct sum of finitely many Prüfer P-submodules.

Proof. The module $A$ is Artinian as a $D$-module by Lemma 6, so $A=B \oplus C$, where $B$ is a direct sum of finitely many Prüfer $P$-submodules and $C$ is a f.g. $D$-submodule. Clearly $B$ is a $D G$-submodule, so there exists a $D G$-submodule $K$ with $A=B \oplus K$ by Lemma 5 .

Since $K \cong{ }_{D} C$, the module $K$ is finitely generated as a $D$-submodule.

Lemma 7. Let $D G$ be the group ring of a finite group $G$ over a Dedekind domain $D$. Let $B$ be a $D G$-submodule of a DG-module $A$ which satisfies the following conditions:

(i) $B=\oplus_{\lambda \in \Lambda} B_{\lambda}$ where $B_{\lambda}$ is a simple $D G$-submodule for all $\lambda \in \Lambda$;

(ii) $A / B$ is a simple $D G$-module and $A / B \approx_{D G} B_{\lambda}$ for all $\lambda \in \Lambda$;

(iii) $\operatorname{Ann}_{D}(B)=\operatorname{Ann}_{D}(A / B)=P$ is a maximal ideal of $D$;

(iv) $\operatorname{char}(D / P)=0$.

Then there exists a $D G$-submodule $K$ such that $A=B \oplus K$.

Proof. Let us fix $y \in P \backslash P^{2}$. Consider a map $f: A \rightarrow A$ such that $f(a)=y a$ for $a \in A$. The map $f$ is a $D G$-endomorphism and $y A=\operatorname{Im}(f) \leq B$. Indeed, $A n n_{D}(A / B)=P$ by (iii), $y \in P \backslash P^{2} \subset P$ and $y \in A n n_{D}(A / B)$, so $y A \leq B$.

If $y A \neq\langle 0\rangle$, then $\operatorname{Ker}(f)=B$ by condition (ii) and $y A=\operatorname{Im}(f) \cong{ }_{D G} A / \operatorname{Ker}(f)=A / B$, so that $y A$ is a simple $D G$-submodule of $B$. Thus $y A \cong_{D G} B_{\lambda}$ for some $\lambda \in \Lambda$ (see [13, Corollary 4.4]), which contradicts condition (ii). Consequently, $y A=\langle 0\rangle$ and $P A=\langle 0\rangle$. It follows that $A$ is a vector space over $D / P$ and $A=B \oplus C$ for a $D$-submodule $C$ by Lemma 5 .

Corollary 5. Let $D G$ be a group ring of a finite group $G$ over a Dedekind domain $D$. Let $A$ be a DG-module such that the following conditions hold:

(i) $\operatorname{sr}_{D G}(A)=r \in \mathbb{N}$;

(ii) $A$ is a $P$-module for some maximal ideal $P$ of $D$;

(iii) $A$ is a f.d. D-module.

If $\operatorname{char}(D / P)=0$, then $A=\oplus_{1 \leq j \leq n} L_{j}$ in which each $L_{j}$ is a $(G, P)$-homogeneous $D G$ submodule of $A$. 
Proof. As before, the $(D / P) G$-module $\Omega_{[P, 1]}(A)$ is a semisimple $D G$-submodule by Lemma 1 and Theorem 11. Consequently, $\Omega_{[P, 1]}(A)=\oplus_{1 \leq j \leq n} H_{j}$ in which each $H_{j}$ is a homogeneous component of $\Omega_{[P, 1]}(A)$ and $A=\oplus_{1 \leq j \leq n} L_{j}$ in which each $L_{j}$ is a $(G, P)$-homogeneous $D G$ submodule of $A$ such that $\Omega_{[P, 1]}\left(L_{j}\right)=H_{j}$.

Corollary 6. Let $D G$ be the group ring of a finite group $G$ over a Dedekind domain D. Let $A$ be a $D G$-module such that the following conditions hold:

(i) $\operatorname{sr}_{D G}(A)=r \in \mathbb{N}$;

(ii) $A$ is a $P$-module for some maximal ideal $P$ of $D$;

If $\operatorname{char}(D / P)=0$, then $A=\oplus_{1 \leq j \leq n} L_{j}$ in which each $L_{j}$ is a $(G, P)$-homogeneous $D G$ submodule of $A$.

Proof. Since $A$ is a $P$-module, $A=\cup_{j \in \mathbb{N}} \Omega_{[P, j]}(A)$. Using induction and Corollary 5 we get the result.

Let $A$ be a $D$-module over a Dedekind domain $D$. The intersection $\Phi(A)$ of all maximal $D$ submodules of $A$ is called the Frattini submodule of $A$. Of course, if $A$ does not include proper maximal submodules, then $\Phi(A)=A$.

If $A$ is a f.g. periodic module and $\left\{a_{j}+\Phi(A) \mid 1 \leq j \leq n\right\}$ is a generating set for $A / \Phi(A)$, then $\left\{a_{j} \mid 1 \leq j \leq n\right\}$ is a generating set for $A$. Moreover, if $\left\{a_{j}+\Phi(A) \mid 1 \leq j \leq n\right\}$ is a minimal generating set for $A / \Phi(A)$, then $\left\{a_{j} \mid 1 \leq j \leq n\right\}$ is a minimal generating set for $A$.

Lemma 8. Let $D G$ be the group ring of a finite group $G$ over a Dedekind domain $D$. Let $A$ be a $D G$-module such that the following conditions hold:

(i) A is a P-module for some maximal ideal $P$ of $D$;

(ii) $A$ is finitely generated as a D-module;

(iii) $A$ is $(G, P)$-homogeneous;

(iv) $\operatorname{char}(D / P)=0$.

The number $\operatorname{sr}_{D G}(A)$ is equal to $r \in \mathbb{N}$ if and only if $\Omega_{[P, 1]}(A)$ is a direct sum of $r$ copies of simple DG-submodules.

Proof. If $\operatorname{sr}_{D G}(A)=r \in \mathbb{N}$, then $\Omega_{[P, 1]}(A)=M_{1} \oplus \cdots \oplus M_{k}$, in which each $M_{j}$ is a simple $D G$-submodule and $M_{j} \cong_{D G} M_{m}$ for all $j, m$, and $k \leq r$ by Lemma 1 and Theorem 1. Note that $\Omega_{[P, 1]}(A) \cong_{D G} A / \Phi(A)$, so $k=r$. Conversely, if $k=r$, then $\operatorname{sr}_{D G}(A)=r \in \mathbb{N}$.

Corollary 7. Let $D G$ be the group ring of a finite group $G$ over a Dedekind domain $D$. Let $A$ be a $D G$-module such that the following conditions hold:

(i) $A$ is a $P$-module for some maximal ideal $P$ of $D$;

(ii) $A$ is finitely generated as a D-module;

(iii) $\operatorname{char}(D / P)=0$.

Then $\operatorname{sr}_{D G}(A)=r \in \mathbb{N}$ if and only if the following conditions hold:

(a) $A=\oplus_{1 \leq j \leq n} H_{j}$ in which each $H_{j}$ is a $(G, P)$-homogeneous DG-submodule of $A$;

(b) $\Omega_{[P, 1]}\left(H_{j}\right)$ is a direct sum of at most $r$ simple $D G$-submodules of $A$;

(c) there exists $t \in \mathbb{N}$ such that $\Omega_{[P, 1]}\left(H_{t}\right)$ is a direct sum of $r$ simple $D G$-submodules of $A$.

Proof. For the proof it is sufficient to use the arguments from the proof of Theorem 1 and the isomorphism $\Omega_{[P, 1]}(A) \cong_{D G} A / \Phi(A)$.

Corollary 8. Let $D G$ be the group ring of a finite group $G$ over a Dedekind domain $D$. Let $A$ be a $D G$-module such that the following conditions hold: 
(i) $A$ is a $P$-module for some maximal ideal $P$ of $D$;

(ii) $\operatorname{char}(D / P)=0$.

The number $\operatorname{sr}_{D G}(A)$ is finite and equals to $r \in \mathbb{N}$ if and only if the following conditions hold:

(a) $A=\oplus_{1 \leq j \leq n} H_{j}$ in which each $H_{j}$ is a $(G, P)$-homogeneous DG-submodule of $A$;

(b) $\Omega_{[P, 1]}\left(H_{j}\right)$ is a direct sum of at most $r$ simple $D G$-submodules of $A$;

(c) there exists $t \in \mathbb{N}$ such that $\Omega_{[P, 1]}\left(H_{t}\right)$ is a direct sum of $r$ simple DG-submodules of $A$.

In particular, if $A$ has finite special rank, then $A$ is Artinian as a D-module.

Corollary 9. Let $D G$ be the group ring of a finite group $G$ over a Dedekind domain $D$. Let $A$ be a $D G$-module which satisfies the following conditions:

(i) $A$ is a D-periodic module;

(ii) $A$ is finitely generated as a D-module;

(iii) $\operatorname{char}(D / P)=0$ for all maximal ideal $P \in \operatorname{Ass}_{R}(A)$.

The number $\operatorname{sr}_{D G}(A)=r \in \mathbb{N}$ if and only if the following conditions hold:

(a) $A=\oplus_{1 \leq j \leq n ; P \in \pi} H_{j, P}$ in which each $H_{j, P}$ is a $(G, P)$-homogeneous DG-submodule of $A$ and $P \in \pi=\operatorname{Ass}_{R}(A)$;

(b) $\Omega_{[P, 1]}\left(H_{j, P}\right)$ is a direct sum of at most $r$ simple $D G$-submodules of $A$;

(c) there exists $t \in \mathbb{N}$ and a maximal ideal $P$ such that $\Omega_{[P, 1]}\left(H_{t, P}\right)$ is a direct sum of $r$ simple $D G$-submodules of $A$.

Proof. Since $A$ is $D$-periodic, $A=\oplus_{P \in \pi} A_{P}$ in which $\pi=\operatorname{Ass}_{R}(A)$ and $A_{P}$ is a $P$-component of $A$. Since $A$ is a f.g. module, the set $\pi$ is finite (see [14, Theorem 7.8]). Now we can apply Corollary 7 .

Proof of the Theorem 3. Since $A$ is $D$-periodic, $A=\oplus_{P \in \pi} A_{P}$ where $\pi=\operatorname{Ass}_{R}(A)$ and $A_{P}$ is a $P$-component of $A$ (see [14, Corollary 3.8]). Now using Corollary 8 we obtain that $A_{P}$ satisfies (ii), (iii) and (iv). Conversely, suppose that $A$ satisfies conditions (i)- (iv). If $B$ is a f.g. $D G$ submodule of $A$, then $B$ has special rank at most $r$ by Corollary 8 , Consequently, $A$ has special rank $r$ by (iv) and Corollary 2 ,

\section{Funding}

This research was supported by the UAEU UPAR Grant G00002160.

\section{REFERENCES}

[1] S. D. Berman. On the theory of representations of finite groups. Doklady Akad. Nauk SSSR (N.S.), 86:885$888,1952$.

[2] S. D. Berman. The number of irreducible representations of a finite group over an arbitrary field. Dokl. Akad. Nauk SSSR (N.S.), 106:767-769, 1956.

[3] A. A. Bovdi. Group rings. (Russian). Kiev.UMK VO, page 155, 1988.

[4] R. Brauer. Zur Darstellungstheorie der Gruppen endlicher Ordnung. Math. Z., 63:406-444, 1956.

[5] I. S. Cohen. Commutative rings with restricted minimum condition. Duke Math. J., 17:27-42, 1950.

[6] P. M. Cohn. Algebra. Vol. 3. pages xii+474, 1991.

[7] C. W. Curtis and I. Reiner. Methods of representation theory. Vol. I. Wiley Classics Library. John Wiley \& Sons, Inc., New York, 1990. With applications to finite groups and orders, Reprint of the 1981 original, A Wiley-Interscience Publication.

[8] M. R. Dixon. Certain rank conditions on groups. Note Mat., 28(suppl. 2):155-180 (2009), 2008.

[9] M. R. Dixon, L. A. Kurdachenko, and I. Y. Subbotin. Ranks of groups. John Wiley \& Sons, Inc., Hoboken, NJ, 2017. The tools, characteristics, and restrictions. 
[10] R. Gilmer. Two constructions of Prüfer domains. J. Reine Angew. Math., 239/240:153-162, 1969.

[11] R. Gilmer. On commutative rings of finite rank. Duke Math. J., 39:381-383, 1972.

[12] R. Gilmer. The n-generator property for commutative rings. Proc. Amer. Math. Soc., 38:477-482, 1973.

[13] L. A. Kurdachenko, J. Otal, and I. Y. Subbotin. Artinian modules over group rings. Frontiers in Mathematics. Birkhäuser Verlag, Basel, 2007.

[14] L. A. Kurdachenko, N. N. Semko, and I. Y. Subbotin. Insight into modules over Dedekind domains, volume 75 of Pratsi Institutu Matematiki Natsional'noi Akademii Nauk Ukraini. Matematika ta jiji Zastosuvannya [Proceedings of Institute of Mathematics of NAS of Ukraine. Mathematics and its Applications]. Natsional'na Akademiya Nauk Ukraini, Institut Matematiki, Kiev, 2008.

[15] A. I. Mal'cev. On groups of finite rank. Mat. Sbornik N.S., 22(64):351-352, 1948.

[16] E. Witt. Die algebraische Struktur des Gruppenringes einer endlichen Gruppe über einem Zahlkörper. J. Reine Angew. Math., 190:231-245, 1952.

UAEU, Al Ain, United Arab Emirates

Email address: vbovdi@gmail.com

Department of Algebra and Geometry, School of Mathematics and Mechanics, National University of DNiPRo, Ukraine

Email address: lkurdachenko@i.ua 